TRANSACTIONS OF THE

AMERICAN MATHEMATICAL SOCIETY

Volume 359, Number 11, November 2007, Pages 5193-5206

S 0002-9947(07)04181-5

Article electronically published on June 22, 2007

\title{
A PRIORI ESTIMATES FOR SECOND ORDER OPERATORS WITH SYMPLECTIC CHARACTERISTIC MANIFOLD
}

\author{
LIDIA MANICCIA AND MARCO MUGHETTI
}

\begin{abstract}
We prove Fefferman's SAK Principle for a class of classical pseudodifferential operators on $\mathbb{R}^{n}$ with symplectic characteristic manifold.
\end{abstract}

\section{INTRODUCTION}

In this paper we are interested in finding sufficient conditions on two second order pseudodifferential operators $P, Q$ on $\mathbb{R}^{n}$ yielding the following inequality:

$$
C_{\epsilon, K}\|Q u\|_{0}^{2} \leq\|P u\|_{0}^{2}+\|u\|_{\epsilon}^{2}, \quad \forall u \in C_{0}^{\infty}(K) .
$$

Here $\epsilon>0$ is a constant, $K$ is a compact set of $\mathbb{R}^{n},\|\cdot\|_{t}$ is the usual norm in the Sobolev space $H^{t}\left(\mathbb{R}^{n}\right), t \in \mathbb{R}$, and $C_{\epsilon, K}$ is a positive constant depending on $\epsilon, K$ (and obviously on $P$ and $Q$ ).

Since every second order operator is continuous from $H_{\text {comp }}^{2}\left(\mathbb{R}^{n}\right)$ to $L_{\mathrm{loc}}^{2}\left(\mathbb{R}^{n}\right)$, inequality (1.1) is trivial whenever $\epsilon \geq 2$, while it is "meaningful" as $\epsilon$ is near zero. Upon considering the fourth order operator $P^{*} P-C_{\epsilon, K} Q^{*} Q$, inequality (1.1) can be restated in terms of the associated quadratic form as follows

$$
\left(\left(P^{*} P-C_{\epsilon, K} Q^{*} Q\right) u, u\right) \geq-\|u\|_{\epsilon}^{2}, \quad \forall u \in C_{0}^{\infty}(K),
$$

where $(\cdot, \cdot)$ denotes the usual $L^{2}\left(\mathbb{R}^{n}\right)$-product.

In [3], Fefferman and Phong proved that a formally self-adjoint pseudodifferential operator $A \in \mathrm{OP} S^{m}\left(\mathbb{R}^{n}\right)$ satisfies

$$
(A u, u) \geq-C_{K}\|u\|_{\frac{m}{2}-1}^{2}, \quad \forall u \in C_{0}^{\infty}(K),
$$

if its Weyl symbol, $a \in S^{m}\left(\mathbb{R}^{2 n}\right)$, is real nonnegative. In what follows, if $s \in$ $S^{m}\left(\mathbb{R}^{2 n}\right)$, its usual Weyl quantization is denoted by $s^{w}(x, D)$ (or simply $s^{w}$ ); see [8] (we also refer to this text for the unexplained notation used throughout).

From a different point of view, Hörmander proved in [7 the same inequality for classical pseudodifferential operators with symbols that can be negative in some directions, by requiring suitable assumptions on the geometry of their characteristic set.

In 17. Tataru provided a new approach based on the FBI transform that allows one to extend inequality (1.3) to classes of symbols with limited smoothness.

Let us now see that an inequality of the kind (1.2), with $\epsilon=3 / 2$, can be readily obtained by using the Fefferman-Phong Inequality (1.3). To this aim, note that the

Received by the editors May 24, 2005.

2000 Mathematics Subject Classification. Primary 35B45; Secondary 35S05.

(C)2007 American Mathematical Society Reverts to public domain 28 years from publication 
Weyl symbol of $P^{*} P-Q^{*} Q$ is

$$
\sigma\left(P^{*} P-Q^{*} Q\right)=\overline{p(x, \xi)} p(x, \xi)-\overline{q(x, \xi)} q(x, \xi)+b(x, \xi),
$$

with $b(x, \xi) \in S^{3}\left(\mathbb{R}^{2 n}\right)$ real, and that the error term $B=b^{w}$ can be easily estimated by the Cauchy-Schwarz Inequality, yielding

$$
|(B u, u)| \leq C_{K}^{\prime}\|u\|_{\frac{3}{2}}^{2}, \quad \forall u \in C_{0}^{\infty}(K) .
$$

Therefore, if $\overline{p(x, \xi)} p(x, \xi)-\overline{q(x, \xi)} q(x, \xi) \geq 0$, i.e. $|p(x, \xi)| \geq|q(x, \xi)|$, we can apply (1.3) to $A=(\bar{p} p-\bar{q} q)^{w}$ and get

$$
\left(\left(P^{*} P-Q^{*} Q\right) u, u\right) \geq-C_{K}^{\prime \prime}\|u\|_{\frac{3}{2}}^{2}, \quad \forall u \in C_{0}^{\infty}(K) .
$$

If we furthermore assume that $P^{*}=P$ and $Q^{*}=Q$ (i.e. $p(x, \xi), q(x, \xi)$ are real), (1.4) holds with $b \in S^{2}\left(\mathbb{R}^{2 n}\right)$, and an application of the Fefferman-Phong Inequality (1.3) in its full strength gives the following improvement:

$$
\left(\left(P^{2}-Q^{2}\right) u, u\right) \geq-C_{K}^{\prime \prime \prime}\|u\|_{1}^{2}, \quad \forall u \in C_{0}^{\infty}(K) .
$$

Actually, in [4] Fefferman conjectured that, under the hypothesis $|p(x, \xi)| \geq$ $|q(x, \xi)|$, a better lower bound for $P^{*} P-Q^{*} Q$ can be obtained. More precisely, Fefferman suggests that if

$$
p(x, \xi) \geq 0 \quad \text { and } \quad|q(x, \xi)| \leq p(x, \xi),
$$

then inequality (1.1) holds for every constant $\epsilon>0$.

In [6] Hérau proved this conjecture when $P$ and $Q$ are pseudodifferential operators in one variable (i.e. $n=1$ ).

In [17] Tataru used his refinement of the Fefferman-Phong Inequality to show that

$$
\left\|b^{w}(x, D) u\right\|_{0}^{2} \leq C\left(\sum_{j=1}^{N}\left\|a_{j}^{w}(x, D) u\right\|_{0}^{2}+\|u\|_{0}^{2}\right)
$$

holds for first order real symbols $b(x, \xi), a_{1}(x, \xi), \ldots, a_{N}(x, \xi)$ with "low regularity" in the $x$-variables (in the class $\mathrm{C}^{1,1} S^{1}$ ), satisfying $|b(x, \xi)| \leq \sum_{j=1}^{N}\left|a_{j}(x, \xi)\right|$.

In this paper we prove Fefferman's conjecture, with $\epsilon=0$, for a special class of second order classical operators. This class is "natural" if we deal with a differential operator $P=p^{w}$ as explained below.

Let $P=p^{w} \in \mathrm{OP} S^{2}\left(\mathbb{R}^{n}\right)$ be a classical pseudodifferential operator with real Weyl symbol $p \sim \sum_{j \geq 0} p_{2-j}$ and smooth characteristic manifold $\Sigma=\{(x, \xi) \in$ $\left.T^{*} \mathbb{R}^{n} \backslash 0: p_{2}(x, \xi)=0\right\}$. If we suppose $p(x, \xi)$ is nonnegative, it readily follows that $p_{2}(x, \xi) \geq 0$, whence $p_{2}$ vanishes on $\Sigma$ to second order, i.e 1

$$
p_{2}(x, \xi) \lesssim|\xi|^{2} \operatorname{dist}_{\Sigma}(x, \xi)^{2}
$$

(dist ${ }_{\Sigma}(x, \xi)$ being the distance of $(x, \xi /|\xi|)$ to $\Sigma$ ). In general, $p_{2}$ does not vanish exactly to second order on $\Sigma$ (i.e. the inequality $p_{2}(x, \xi) \gtrsim|\xi|^{2} \operatorname{dist}_{\Sigma}(x, \xi)^{2}$ does not hold) and (1.6) is too weak to control the behavior of $p_{2}$ near $\Sigma$. As a trial to attack Fefferman's conjecture, from now on we assume that $\Sigma$ is a symplectic manifold given by the transversal intersection of two smooth closed cones $\Sigma_{1}, \Sigma_{2}$

\footnotetext{
${ }^{1}$ From now on, for two nonnegative functions $f, g$ on $\mathbb{R}^{2 n}$ we write $f \lesssim g$ (respectively $f \gtrsim g$ ) when there exists a constant $c>0$ such that $f(x, \xi) \leq c g(x, \xi)$ (respectively $f(x, \xi) \geq c g(x, \xi)$ ). We simply write $f \approx g$ when $f \lesssim g$ and $f \gtrsim g$.
} 
of codimension 1 . In order to fix how $p_{2}$ vanishes at $\Sigma$, we finally suppose that, for a positive integer $h$,

$$
p_{2}(x, \xi) \approx|\xi|^{2}\left(\operatorname{dist}_{\Sigma_{1}}(x, \xi)^{2 h}+\operatorname{dist}_{\Sigma_{2}}(x, \xi)^{2}\right) .
$$

Note that if $h=1$, then $p_{2}$ vanishes exactly to second order on $\Sigma$, but this is, in general, not the case when $h>1$. As regards the subprincipal symbol $p_{1}$ of $P$, let us first consider the case where $P$ is a differential operator of second order. We start by observing that the nonnegativity of the total symbol $p(x, \xi)$ forces the subprincipal symbol $p_{1}$ to vanish on $\Sigma$.

Indeed, for any $\rho=(\bar{x}, \bar{\xi}) \in \Sigma$ one has, in view of the homogeneity, $p_{1}(\bar{x}, \bar{\xi}) \geq 0$. Since $P$ is a differential operator, $\Sigma$ is invariant under the action of the antipodal map $(x, \xi) \rightarrow(x,-\xi)$ and, again from the homogeneity, it follows that $0 \leq$ $p_{1}(\bar{x},-\bar{\xi})=-p_{1}(\bar{x}, \bar{\xi})$, whence $p_{1}(\bar{x}, \bar{\xi})=0$, as claimed.

Furthermore, in the case of differential operators it is "natural" to assume that $\Sigma_{1}$ and $\Sigma_{2}$ are invariant under the action of the antipodal map. As a consequence, conditions (1.5) and (1.7) impose a stricter behavior on $p_{1}$ near $\Sigma$, namely

$$
\left|p_{1}(x, \xi)\right| \lesssim|\xi|\left(\operatorname{dist}_{\Sigma_{1}}(x, \xi)^{h}+\operatorname{dist}_{\Sigma_{2}}(x, \xi)\right) .
$$

In fact, the structure of the characteristic manifold $\Sigma$ allows us to reduce the proof of (1.8) to the flat case, i.e. when $p$ is a classical symbol and $\Sigma_{1}=\{(x, \xi) \in$ $\left.T^{*} \mathbb{R}^{n} \backslash 0: x_{1}=0\right\}, \Sigma_{2}=\left\{(x, \xi) \in T^{*} \mathbb{R}^{n} \backslash 0: \xi_{1}=0\right\}$ (see Proposition 2.3). Moreover, we can also assume that $p=p_{2}+p_{1}+p_{0}$ with $p_{j} \in S^{j}\left(\mathbb{R}^{2 n}\right)$ such that $p_{j}(x, t \xi)=t^{j} p_{j}(x, \xi), j=0,1,2$, for any $0 \neq t \in \mathbb{R}$ (see Remark 2.4). Note that, in the flat case, one has $\operatorname{dist}_{\Sigma_{1}}(x, \xi) \approx\left|x_{1}\right|$ and $\operatorname{dist}_{\Sigma_{2}}(x, \xi) \approx\left|\xi_{1}\right| /|\xi|$.

Since $p_{1}=0$ on $\Sigma=\Sigma_{1} \cap \Sigma_{2}$, we can use Taylor's expansion to get

$$
p_{1}(x, \xi)=\alpha_{0}(x, \xi) \xi_{1}+\beta_{0}(x, \xi) x_{1}
$$

with $\alpha_{0}, \beta_{0}$ homogeneous of degree 0 and 1 respectively (i.e. $\alpha_{0}(x, t \xi)=\alpha_{0}(x, \xi)$, $\beta_{0}(x, t \xi)=t \beta_{0}(x, \xi)$ for every $\left.t \in \mathbb{R}, t \neq 0\right)$. We now show that $\beta_{0}=0$ on $\Sigma$. To this purpose, by using the expression (1.9) for the subprincipal symbol, we evaluate $p\left(x, 0, t \xi^{\prime}\right)$ for $t \neq 0\left(\xi=\left(\xi_{1}, \xi^{\prime}\right) \in \mathbb{R} \times \mathbb{R}^{n-1}\right)$ and then use (1.7) to get, by means of the homogeneity properties of the terms,

$$
C x_{1}^{2 h}\left|\xi^{\prime}\right|^{2} t^{2}+x_{1} \beta_{0}\left(x, 0, \xi^{\prime}\right) t+p_{0}\left(x, 0, \xi^{\prime}\right) \geq 0, \quad C>0, \forall t \neq 0 .
$$

Therefore, it follows that $\beta_{0}\left(x, 0, \xi^{\prime}\right)^{2} \leq 4 C x_{1}^{2 h-2}\left|\xi^{\prime}\right|^{2} p_{0}\left(x, 0, \xi^{\prime}\right)$, whence $\beta_{0}=0$ on $\Sigma$. Applying Taylor's formula once more yields $\beta_{0}(x, \xi)=\tilde{\alpha}_{1}(x, \xi) \xi_{1}+\beta_{1}(x, \xi) x_{1}$, where $\tilde{\alpha}_{1}$ and $\beta_{1}$ are homogeneous of degree 0 and 1 respectively. Hence

$$
p_{1}(x, \xi)=\left(\alpha_{0}(x, \xi)+x_{1} \tilde{\alpha}_{1}(x, \xi)\right) \xi_{1}+\beta_{1}(x, \xi) x_{1}^{2} .
$$

An iteration of the above procedure leads to

$$
p_{1}(x, \xi)=\alpha(x, \xi) \xi_{1}+\beta(x, \xi) x_{1}^{h}
$$

which shows the precise structure of $p_{1}$ and, in particular, gives (1.8).

The above arguments suggest considering second order classical pseudodifferential operators satisfying (1.7) and (1.8). It is worth noting that these conditions give rise to an invariant operator class as shown in Remark 3.6 .

We are now ready to state the main theorem of this paper.

Theorem 1.1. Let $\Sigma$ be a symplectic manifold given by the transversal intersection of two smooth cones $\Sigma_{1}$ and $\Sigma_{2}$ of codimension 1. Consider $p$ and $q$ classical symbols in $S^{2}\left(\mathbb{R}^{2 n}\right)$ with $p \geq 0$ having characteristic manifold $\Sigma$ and satisfying (1.7), (1.8). 
Suppose that

$$
|q(x, \xi)| \leq p(x, \xi), \quad \forall(x, \xi) \in \mathbb{R}^{2 n} .
$$

Then, for every compact set $K \subset \subset \mathbb{R}^{n}$, there exists $C>0$ such that

$$
\left\|q^{w} u\right\|_{0}^{2} \leq C\left(\left\|p^{w} u\right\|_{0}^{2}+\|u\|_{0}^{2}\right), \quad \forall u \in C_{0}^{\infty}(K) .
$$

We point out that this theorem holds true also if $\Sigma_{1}$ and $\Sigma_{2}$ are involutive cones of codimension $\nu>1$ and the proof can be easily adapted from the case $\nu=1$. We refer to Section 3 for further details.

The paper is organized as follows. In Section 2 we show that (1.11) is equivalent to a finite number of microlocal estimates, and in Section 3 we prove Theorem 1.1 . Here we use suitable Fourier Integral Operators (FIO) to reduce the proof to the flat case $p_{2}(x, \xi) \approx \xi_{1}^{2}+|\xi|^{2} x_{1}^{2 h}$. A crucial point is the application of Theorems 4.1 and 4.3 of Section 4 which are direct consequences of the arguments contained in [1] even if they are not explicitly stated there. The final Section 4 is a technical appendix, where we discuss the adjustments required in [11] to prove these theorems.

\section{Reduction to micRolocal estimates}

In this section we prove that inequality (1.11) can be reduced to a finite number of microlocal estimates "supported" in conic regions of $T^{*} \mathbb{R}^{n} \backslash 0$. Let $K \subset \mathbb{R}^{n}$ be the compact set in Theorem 1.1, and choose a bounded open set $\Omega$ of $\mathbb{R}^{n}$ with $K \subset \Omega$. Fix a finite family $\left\{O_{j}\right\}_{j=0,1, \ldots, N}$ of open conic sets of $T^{*} \Omega \backslash 0$, such that $\bigcup_{j=0}^{N} O_{j}=T^{*} \Omega \backslash 0$.

Finally, consider two families $\left\{\varphi_{j}\right\}_{j=0,1, \ldots, N},\left\{\psi_{j}\right\}_{j=0,1, \ldots, N}$ of positively homogeneous symbols in $S^{0}\left(\mathbb{R}^{2 n}\right)$, subordinated to the covering $\left\{O_{j}\right\}_{j=0,1, \ldots, N}$, such that $0 \leq \psi_{j}, \varphi_{j} \leq 1, \psi_{j} \varphi_{j}=\varphi_{j}$ and $\sum_{j=0}^{N} \varphi_{j}^{2}=1$ in a conic neighborhood $W$ of $\Pi^{-1}(K)$ ( $\Pi$ denotes the canonical projection $T^{*} \mathbb{R}^{n} \backslash 0 \ni(x, \xi) \longmapsto x \in \mathbb{R}^{n}$ ). As usual, $s \in S^{m}\left(\mathbb{R}^{2 n}\right)$ is called a positively homogeneous symbol if

$$
s(x, t \xi)=t^{m} s(x, \xi), \quad \forall(x, \xi) \in \mathbb{R}^{2 n},|\xi| \gtrsim 1 \text { and } \forall t \in \mathbb{R}, t \gtrsim 1 .
$$

From now on, without loss of generality, we suppose that $p$ and $q$ are supported in the neighborhood $W$. This is a consequence of the following remark.

Remark 2.1. Fix $\gamma \in C_{0}^{\infty}\left(\mathbb{R}^{n}\right)$ such that $\gamma=1$ on a neighborhood of $K$ and consider $s \in S^{2}\left(\mathbb{R}^{2 n}\right)$. Then there exist positive constants $c_{1}$ and $c_{2}$ such that

$$
c_{1}\left\|s^{w} u\right\|_{0}-\frac{1}{c_{1}}\|u\|_{0} \leq\left\|(s \gamma)^{w} u\right\|_{0} \leq c_{2}\left(\left\|s^{w} u\right\|_{0}+\|u\|_{0}\right), \quad \forall u \in C_{0}^{\infty}(K) .
$$

We are now ready to prove the microlocal reduction mentioned above. We point out that this does not require either hypotheses (1.7) and (1.8) or any geometrical assumptions on $\Sigma$, but only that $p, q \in S^{2}\left(\mathbb{R}^{2 n}\right)$ with $p$ real nonnegative.

Proposition 2.2. Let $p, q \in S^{2}\left(\mathbb{R}^{2 n}\right)$ be supported in the conic neighborhood $W$ of $\Pi^{-1}(K)$ with $p$ real nonnegative. Then inequality (1.11) is satisfied if the following estimates hold:

$$
\left\|\left(\psi_{j}^{2} q\right)^{w} \varphi_{j}^{w} u\right\|_{0}^{2} \leq C_{j}\left(\left\|\left(\psi_{j}^{2} p\right)^{w} \varphi_{j}^{w} u\right\|_{0}^{2}+\|u\|_{0}^{2}\right), \quad \forall u \in C_{0}^{\infty}(K), j=0, \ldots, N,
$$

where $C_{j}$ are positive constants. 
Proof. Since $\psi_{j} \varphi_{j}=\varphi_{j}$, the composition formula in Theorem 18.5.4 of [8] yields, for every $j=0, \ldots, N$,

$$
\varphi_{j}^{w}\left(\psi_{j}^{2} q\right)^{w} \varphi_{j}^{w}=\left(q \varphi_{j}^{2}+r_{j}\right)^{w}, \quad r_{j} \in S^{0}\left(\mathbb{R}^{2 n}\right),
$$

whence, in view of the support property of $q$, we get

$$
\sum_{j=0}^{N} \varphi_{j}^{w}\left(\psi_{j}^{2} q\right)^{w} \varphi_{j}^{w}=\left(q \sum_{j=0}^{N} \varphi_{j}^{2}\right)^{w}+\sum_{j=0}^{N} r_{j}^{w}=q^{w}+r^{w},
$$

where $r=\sum_{j=0}^{N} r_{j} \in S^{0}\left(\mathbb{R}^{2 n}\right)$. We thus obtain

$$
\left\|q^{w} u\right\|_{0}^{2}=\sum_{j=0}^{N}\left(\left(\psi_{j}^{2} q\right)^{w} \varphi_{j}^{w} u, \varphi_{j}^{w} q^{w} u\right)-\left(r^{w} u, q^{w} u\right), \quad \forall u \in C_{0}^{\infty}(K) .
$$

Furthermore, there are suitable positive constants $c$ and $c_{j}$, such that, for every given $\epsilon \in(0,1)$, one has

$$
\left|\left(r^{w} u, q^{w} u\right)\right| \leq \frac{1}{\epsilon^{2}}\left\|r^{w} u\right\|_{0}^{2}+\epsilon^{2}\left\|q^{w} u\right\|_{0}^{2} \leq \epsilon^{2}\left\|q^{w} u\right\|_{0}^{2}+c / \epsilon^{2}\|u\|_{0}^{2}, \quad \forall u \in C_{0}^{\infty}(K),
$$

and

$$
\left|\left(\left(\psi_{j}^{2} q\right)^{w} \varphi_{j}^{w} u, \varphi_{j}^{w} q^{w} u\right)\right| \leq \frac{1}{\epsilon^{2}}\left\|\left(\psi_{j}^{2} q\right)^{w} \varphi_{j}^{w} u\right\|_{0}^{2}+c_{j} \epsilon^{2}\left\|q^{w} u\right\|_{0}^{2}, \quad \forall u \in C_{0}^{\infty}(K) .
$$

From (2.2), one then gets

$$
\epsilon^{2}\left(1-\epsilon^{2}\left(1+\sum_{j=0}^{N} c_{j}\right)\right)\left\|q^{w} u\right\|_{0}^{2} \leq \sum_{j=0}^{N}\left\|\left(\psi_{j}^{2} q\right)^{w} \varphi_{j}^{w} u\right\|_{0}^{2}+c\|u\|_{0}^{2}, \quad \forall u \in C_{0}^{\infty}(K) .
$$

If we choose $\epsilon \in(0,1)$ small enough in the inequality above, we can hence conclude that

$$
\left\|q^{w} u\right\|_{0}^{2} \lesssim \sum_{j=0}^{N}\left\|\left(\psi_{j}^{2} q\right)^{w} \varphi_{j}^{w} u\right\|_{0}^{2}+\|u\|_{0}^{2}, \quad \forall u \in C_{0}^{\infty}(K), j=0,1, \ldots, N .
$$

To complete the proof, it remains to show the estimate

$$
\sum_{j=0}^{N}\left\|\left(\psi_{j}^{2} p\right)^{w} \varphi_{j}^{w} u\right\|_{0}^{2} \lesssim\left\|p^{w} u\right\|_{0}^{2}+\|u\|_{0}^{2}, \quad \forall u \in C_{0}^{\infty}(K), j=0,1, \ldots, N .
$$

Since $\varphi_{j}^{w}$ is an operator of order 0 and $\varphi_{j}, \psi_{j}^{2}-1$ have disjoint supports, we easily see that, for all $j=0, \ldots, N$ and all $u \in C_{0}^{\infty}(K)$,

$$
\begin{aligned}
\left\|\left(\psi_{j}^{2} p\right)^{w} \varphi_{j}^{w} u\right\|_{0}^{2} & \lesssim\left\|\varphi_{j}^{w} p^{w} u\right\|_{0}^{2}+\left\|\varphi_{j}^{w}\left(\left(\psi_{j}^{2}-1\right) p\right)^{w} u\right\|_{0}^{2}+\left\|\left[\left(\psi_{j}^{2} p\right)^{w}, \varphi_{j}^{w}\right] u\right\|_{0}^{2} \\
& \lesssim\left\|p^{w} u\right\|_{0}^{2}+\left\|\left[\left(\psi_{j}^{2} p\right)^{w}, \varphi_{j}^{w}\right] u\right\|_{0}^{2}+\|u\|_{0}^{2} .
\end{aligned}
$$

Here we denote by $[A, B]$ the usual commutator of two operators $A$ and $B$.

This means that, in order to obtain (2.4), it is enough to prove that

$$
\left\|\left[\left(\psi_{j}^{2} p\right)^{w}, \varphi_{j}^{w}\right] u\right\|_{0}^{2} \lesssim\left\|p^{w} u\right\|_{0}^{2}+\|u\|_{0}^{2}, \quad \forall u \in C_{0}^{\infty}(K) .
$$

By direct computation one has $\sigma\left(\left[\left(\psi_{j}^{2} p\right)^{w}, \varphi_{j}^{w}\right]\right)-\frac{1}{i}\left\{\psi_{j}^{2} p, \varphi_{j}\right\} \in S^{0}\left(\mathbb{R}^{2 n}\right)(\{f, g\}$ is the Poisson bracket of the functions $f$ and $g$ ). Thus, since

$$
\left[\left(\psi_{j}^{2} p\right)^{w}, \varphi_{j}^{w}\right]^{*}\left[\left(\psi_{j}^{2} p\right)^{w}, \varphi_{j}^{w}\right]-\left(\left\{\psi_{j}^{2} p, \varphi_{j}\right\}^{2}\right)^{w}
$$


is an operator of order zero, we have

$$
\left\|\left[\left(\psi_{j}^{2} p\right)^{w}, \varphi_{j}^{w}\right] u\right\|_{0}^{2} \lesssim\left(\left(\left\{\psi_{j}^{2} p, \varphi_{j}\right\}^{2}\right)^{w} u, u\right)+\|u\|_{0}^{2}, \quad \forall u \in C_{0}^{\infty}(K) .
$$

We use the Fefferman-Phong Inequality (1.3) to estimate the right-hand-side of (2.6). To this purpose, we now prove that $\left\{\psi_{j}^{2} p, \varphi_{j}\right\}^{2} \lesssim p$. Recall that, for every nonnegative function $f \in C^{2}(\mathbb{R})$ with bounded second derivative, the following well known inequality holds:

$$
f^{\prime}(t)^{2} \leq 2\left\|f^{\prime \prime}\right\|_{\infty} f(t)
$$

whence $\left(\partial_{\xi_{i}}\left(\psi_{j}^{2} p\right)\right)^{2} \lesssim \psi_{j}^{2} p \lesssim p$ and $|\xi|^{-4}\left(\partial_{x_{i}}\left(\psi_{j}^{2} p\right)\right)^{2} \lesssim \psi_{j}^{2} p|\xi|^{-2} \lesssim p|\xi|^{-2}$. Therefore one gets $\left\{\psi_{j}^{2} p, \varphi_{j}\right\}^{2} \lesssim p$. By means of (1.3) we then obtain

$$
\left(\left(\left\{\psi_{j}^{2} p, \varphi_{j}\right\}^{2}\right)^{w} u, u\right) \lesssim\left(p^{w} u, u\right)+\|u\|_{0}^{2}, \quad \forall u \in C_{0}^{\infty}(K), j=0, \ldots, N .
$$

This, in addition to (2.6), gives (2.5), so that (2.4) is proved and the proof is complete.

The proof of Theorem 1.1] is thus reduced to the proof of the inequalities (2.1) supported in the conic regions $O_{j}$.

To prove these inequalities, we now choose a suitable covering $\left\{O_{j}\right\}_{j=0,1, \ldots, N}$ that takes into account the geometry of the characteristic manifold $\Sigma$ and hypotheses (1.7), (1.8). Roughly speaking, if $O_{j} \cap \Sigma=\emptyset, p$ is elliptic in $O_{j}$ and the related microlocal estimate in (2.1) easily follows, hence the crucial point turns out to be the proof of the estimates for which $O_{j} \cap \Sigma \neq \emptyset$. In this case, we show that there exists a symplectomorphism $\chi$ (canonical flattening) that will allow us, in Section 3. to write $p^{w}$ in a "canonical" form.

More precisely, suitable adjustments of Theorem 21.2.4 [8] (see also Lemma 4.1 [9] and Lemma 6.1 [15]) yield the following proposition.

Proposition 2.3. Let $\Sigma_{1}, \Sigma_{2}, \Sigma$ be as in Theorem 1.1. Then, for every point $\rho$ of $\Sigma$, there exist a conic neighborhood $O_{\rho}$ of $\rho$ in $T^{*} \mathbb{R}^{n} \backslash 0$, a conic neighborhood $O^{\prime}$ in $T^{*} \mathbb{R}^{n} \backslash 0$ and a symplectomorphism (positively homogeneous of degree one in the fibers) $\chi_{\rho}: O_{\rho} \longrightarrow O^{\prime}$ for which $\chi_{\rho}\left(O_{\rho} \cap \Sigma_{1}\right)=\left\{(y, \eta) \in O^{\prime} \mid y_{1}=0\right\}$ and $\chi_{\rho}\left(O_{\rho} \cap \Sigma_{2}\right)=\left\{(y, \eta) \in O^{\prime} \mid \eta_{1}=0\right\}$. Such a map $\chi_{\rho}$ is called a canonical flattening of $\Sigma_{1}$ and $\Sigma_{2}$ in $O_{\rho}$.

Proof. Since $\Sigma$ is a cone, Euler's identity assures that the radial vector $r=\sum \xi_{j} \partial_{\xi_{j}}$ belongs to the tangent space $T_{\rho} \Sigma$ to $\Sigma$ at $\rho \in \Sigma$. On the other hand, $\Sigma$ is a symplectic manifold so that, upon denoting by $\left(T_{\rho} \Sigma\right)^{\sigma}$ the orthogonal space with respect to the canonical 2-form $\sum_{j=1}^{n} d \xi_{j} \wedge d x_{j}$, one has $T_{\rho} \Sigma \cap\left(T_{\rho} \Sigma\right)^{\sigma}=\{0\}$, so that the radial vector $r$ does not belong to $\left(T_{\rho} \Sigma\right)^{\sigma}$.

An application of the Darboux theorem (see [8], Theorem 21.1.9) shows that we can assume $\Sigma_{1}=\left\{(y, \eta): y_{1}=0\right\}$ and $\Sigma_{2}=\{(y, \eta): v(y, \eta)=0\}$. Since $\Sigma=\left\{(y, \eta): y_{1}=v(y, \eta)=0\right\}$ is symplectic, we have $\partial v / \partial \eta_{1}=\left\{v, y_{1}\right\} \neq 0$ at $\rho$. Hence, by the Implicit Function Theorem, there exists a smooth positively homogeneous function $f\left(y, \eta_{2}, \ldots, \eta_{n}\right)$ of degree 1 in the fibers, such that, near $\rho$,

$$
\Sigma_{2}=\left\{(y, \eta): \eta_{1}-f\left(y, \eta_{2}, \ldots, \eta_{n}\right)=0\right\}
$$

Therefore, we now have $\left\{\eta_{1}-f\left(y, \eta_{2}, \ldots, \eta_{n}\right), y_{1}\right\}=1$ conically near $\rho$ and, by applying again the Darboux theorem, we complete the proof. 
Remark 2.4. If the cones $\Sigma_{1}, \Sigma_{2}$ in Proposition 2.3 are invariant under the action of the antipodal map $\mathcal{I}:(x, \xi) \longmapsto(x,-\xi)$, then the canonical flattening $\chi_{\rho}$ can be defined in the "complete" cone $V_{\rho} \cup \mathcal{I}\left(V_{\rho}\right)$ and assumed to be homogeneous, i.e. $\chi_{\rho} \circ \mathcal{I}=\mathcal{I} \circ \chi_{\rho}$ (and not simply positively homogeneous).

In view of Proposition 2.3. we can then choose the family $\left\{O_{j}\right\}_{j=0,1, \ldots, N}$ of open conic sets of $T^{*} \Omega \backslash 0$, considered at the beginning of this section, satisfying the following requirements:

- $\bigcup_{j=0}^{N} O_{j}=T^{*} \Omega \backslash 0, O_{0} \cap \Sigma=\emptyset, \quad \Sigma \cap T^{*} \Omega \subset \bigcup_{j=1}^{N} O_{j} ;$

- for any $j=1, \ldots, N$ there exists a canonical flattening $\chi_{j}$ of $\Sigma_{1}$ and $\Sigma_{2}$ in $O_{j}$.

Since $O_{0} \cap \Sigma=\emptyset$, inequality (2.1) with $j=0$ immediately follows from the ellipticity of $p^{w}$ in the conic set $O_{0}$. Therefore, by Proposition 2.2. the proof of Theorem 1.1 is reduced to the proof of (2.1) with $j=1, \ldots, N$.

\section{Proof of Theorem 1.1}

In this section we use the standard FIO theory (see [8, Vol.IV and [18]) to "reduce" the operator $p^{w}$ to the Grushin operator $D_{1}^{2}+y_{1}^{2 h} \sum_{j=2}^{n} D_{j}^{2}$, so that Theorems 4.1 and 4.3 can be applied in order to prove Theorem 1.1.

More precisely, we construct, for every $j=1, \ldots, N$, two properly supported Fourier integral operators $F_{j}, F_{j}^{*}$ of order 0 , associated with $\chi_{j}, \chi_{j}^{-1}$, such that, possibly after shrinking $O_{j}$, one has, for any symbols $\phi(x, \xi), \theta(y, \eta) \in S^{m}\left(\mathbb{R}^{2 n}\right)$ with supp $\phi \subset O_{j}$ and $\operatorname{supp} \theta \subset \chi_{j}\left(O_{j}\right)$,

$F_{j}^{*} F_{j} \phi^{w}-\phi^{w}, \quad \phi^{w} F_{j}^{*} F_{j}-\phi^{w}, \quad F_{j} F_{j}^{*} \theta^{w}-\theta^{w}, \quad \theta^{w} F_{j} F_{j}^{*}-\theta^{w} \in \mathrm{OP} S^{-\infty}\left(\mathbb{R}^{n}\right)$.

Furthermore, if $A \in \mathrm{OP} S^{m}\left(\mathbb{R}^{n}\right)$ is a classical pseudodifferential operator, then so is $F_{j} A F_{j}^{*} \in \mathrm{OP} S^{m}\left(\mathbb{R}^{n}\right)$ and, upon denoting by $\sigma_{\text {princ }}(B)$ the principal symbol of any classical operator $B$, one has, in $\chi_{j}\left(O_{j}\right)$,

$$
\sigma_{\text {princ }}\left(F_{j} A F_{j}^{*}\right)=\sigma_{\text {princ }}(A) \circ \chi_{j}^{-1} \text {. }
$$

This shows that hypothesis (1.7) is invariant under conjugation by Fourier integral operators, moreover, since $\chi_{j}$ is a canonical flattening, it also gives

$$
\sigma_{\text {princ }}\left(F_{j} p_{2}^{w} F_{j}^{*}\right)=\left(p_{2} \circ \chi_{j}^{-1}\right)(y, \eta) \approx \eta_{1}^{2}+y_{1}^{2 h}|\eta|^{2}, \quad \forall(y, \eta) \in \chi_{j}\left(O_{j}\right) .
$$

Therefore, roughly speaking, we can say that $p_{2}^{w}$ is reduced to a Grushin-type operator.

We now show that, in view of hypotheses (1.8) and (1.10), the symbol of $F_{j} q^{w} F_{j}^{*}$ is dominated by $p_{2} \circ \chi_{j}^{-1}$ in the conic region $\chi_{j}\left(O_{j}\right)$; this will allow us to apply Theorem 4.1 to $F_{j}\left(\psi_{j}^{2} q^{w}\right) F_{j}^{*}$ in order to get (2.1) for every $j=1, \ldots, N$.

Proposition 3.1. Under the hypotheses of Theorem 1.1 we have

$$
\left|\sigma\left(F_{j}\left(\psi_{j}^{2} q\right)^{w} F_{j}^{*}\right)\right| \lesssim\left(\psi_{j}^{2} p_{2}\right) \circ \chi_{j}^{-1}+1, \quad j=1, \ldots, N .
$$

The crucial point in the proof of this proposition is to check that

$$
\sigma\left(F_{j}\left(\psi_{j}^{2} q\right)^{w} F_{j}^{*}\right)=\left(\left(\psi_{j}^{2} q\right) \circ \chi^{-1}\right)(y, \eta)+r(y, \eta),
$$


with $r \in S^{1}\left(\mathbb{R}^{2 n}\right)$ satisfying $|r(y, \eta)| \lesssim\left(\left(\psi_{j}^{2} p_{2}\right) \circ \chi_{j}^{-1}\right)(y, \eta)+1$. To this aim, let us present some preliminary results. Recall that, for any $w \in \mathbb{R}^{n}$ we use the notation $w=\left(w_{1}, w^{\prime}\right)$ with $w^{\prime} \in \mathbb{R}^{n-1}$.

Lemma 3.2. Let $s_{2} \in S^{2}\left(\mathbb{R}^{2 n}\right)$ be a positively homogeneous symbol of degree 2 such that

$$
\left|s_{2}(y, \eta)\right| \lesssim \eta_{1}^{2}+|\eta|^{2} y_{1}^{2 h}, \quad \forall(y, \eta) \in \mathbb{R}^{2 n},|\eta| \gtrsim 1 .
$$

Then there exist symbols $g_{i} \in S^{i}\left(\mathbb{R}^{2 n}\right), i=0,1,2$, positively homogeneous of degree $i$, such that, in any region $\mathcal{R}=\left\{(y, \eta) \in \mathbb{R}^{2 n}:\left|\eta^{\prime}\right| \gtrsim\left|\eta_{1}\right|\right\}$,

$$
s_{2}(y, \eta)=\eta_{1}^{2} g_{0}(y, \eta)+\eta_{1} y_{1}^{h} g_{1}(y, \eta)+y_{1}^{2 h} g_{2}(y, \eta), \quad \forall(y, \eta) \in \mathcal{R},|\eta| \gtrsim 1 .
$$

Proof. First observe that, if $\left|\eta^{\prime}\right|$ is suitably large, $s_{2}\left(0, y^{\prime}, 0, \eta^{\prime}\right)=0$, hence, by Taylor's expansion, we get

$$
s_{2}(y, \eta)=y_{1} \tilde{g}_{2}(y, \eta)+\eta_{1} \tilde{g}_{1}(y, \eta), \quad\left|\eta^{\prime}\right| \gtrsim 1
$$

where $\tilde{g}_{i} \in S^{i}\left(\mathbb{R}^{2 n}\right)$ are positively homogeneous symbols of degree $i=1,2$.

If we consider in (3.7) first $y_{1}=0$ and then $\eta_{1}=0$, in view of (3.5), we get $\tilde{g}_{1}\left(0, y^{\prime}, 0, \eta^{\prime}\right)=0$ and $\tilde{g}_{2}\left(0, y^{\prime}, 0, \eta^{\prime}\right)=0$. Hence, again by Taylor's expansion, we obtain $\tilde{g}_{1}=y_{1} \bar{g}_{1}+\eta_{1} g_{0}$ and $\tilde{g}_{2}=y_{1} g_{2}+\eta_{1} \overline{\bar{g}}_{1}$; thus from (3.7), by setting $g_{1}=\overline{\bar{g}}_{1}+\bar{g}_{1}$, we get (3.6) when $h=1$. In order to treat the case $h \geq 2$, we now show that if for some $2 \leq N \leq h$ we have

$$
s_{2}(y, \eta)=\eta_{1}^{2} f_{0}(y, \eta)+\eta_{1} y_{1}^{N-1} f_{1}(y, \eta)+y_{1}^{2(N-1)} f_{2}(y, \eta), \quad\left|\eta^{\prime}\right| \gtrsim 1,
$$

where $f_{i}$ are symbols in $S^{i}\left(\mathbb{R}^{2 n}\right)$ positively homogeneous of degree $i=0,1,2$, then the same holds with $N$ in place of $N-1$.

By arguing as above, from (3.8) it follows that

$$
s_{2}(y, \eta)=\eta_{1}^{2} f_{0}(y, \eta)+\eta_{1} y_{1}^{N-1} \tilde{f}_{1}(y, \eta)+y_{1}^{2 N} \tilde{f}_{2}(y, \eta), \quad\left|\eta^{\prime}\right| \gtrsim 1 .
$$

By (3.5) and (3.9) one gets

$$
\left|\eta_{1} y_{1}^{N-1} \tilde{f}_{1}(y, \eta)\right| \leq c\left(\eta_{1}^{2}+|\eta|^{2}\left(y_{1}^{2 h}+y_{1}^{2 N}\right)\right), \quad\left|\eta^{\prime}\right| \gtrsim 1,
$$

hence, if we choose $\eta_{1}=y_{1}^{N},\left|y_{1}\right| \leq 1$, we obtain

$$
\left|\tilde{f}_{1}\left(y, y_{1}^{N}, \eta^{\prime}\right)\right| \lesssim\left|y_{1}\right|\left(1+\left|\eta^{\prime}\right|^{2}\right)
$$

Letting $y_{1} \rightarrow 0$ with $\left|\eta^{\prime}\right| \gtrsim 1$ yields $\tilde{f}_{1}\left(0, y^{\prime}, 0, \eta^{\prime}\right)=0$, and the same arguments as before give (3.8) with $N$ instead of $N-1$.

By proceeding as we did in the case $h=1$, we see that (3.8) holds for $N=2$ and this shows that (3.8) holds also for $N=h \geq 2$. Since in the region $\mathcal{R}$ one has $|\eta| \approx\left|\eta^{\prime}\right|$, the proof readily follows.

The following corollary shows that $q$ has a precise structure in view of (1.10).

Assume that, for $j=1, \ldots, N, u_{j} \in S^{0}\left(\mathbb{R}^{2 n}\right)$ and $v_{j} \in S^{1}\left(\mathbb{R}^{2 n}\right)$ are positively homogeneous local equations in $O_{j}$ of $\Sigma_{1}$ and $\Sigma_{2}$, respectively. Moreover, by Proposition [2.3, we can also assume, without loss of generality, that $u_{j} \circ \chi_{j}^{-1}=y_{1}$ and $v_{j} \circ \chi_{j}^{-1}=\eta_{1}, j=1, \ldots, N$. Finally, we can suppose that, for every $(y, \eta) \in \chi_{j}\left(O_{j}\right)$, $j=1, \ldots, N$, one has $\left|\eta^{\prime}\right| \gtrsim\left|\eta_{1}\right|$. 
Corollary 3.3. Let $q \in S^{2}\left(\mathbb{R}^{2 n}\right)$ be as in Theorem 1.1 and denote by $q_{2}$ its principal symbol. Then there exist $a_{i, j} \in S^{i}\left(\mathbb{R}^{2 n}\right), j=1, \ldots, N$, positively homogeneous of degree $i=0,1,2$ such that

$$
\psi_{j}^{2} q_{2}=\left(\psi_{j}^{2} a_{2, j}\right) u_{j}^{2 h}+\left(\psi_{j}^{2} a_{1, j}\right) u_{j}^{h} v_{j}+\left(\psi_{j}^{2} a_{0, j}\right) v_{j}^{2}, \quad \forall(x, \xi) \in \mathbb{R}^{2 n},|\xi| \gtrsim 1 .
$$

Proof. We consider, for $j=1, \ldots, N, \tilde{\psi}_{j} \in S^{0}\left(\mathbb{R}^{2 n}\right)$ supported in $O_{j}$ positively homogeneous of degree 0 , such that $\psi_{j} \tilde{\psi}_{j}=\psi_{j}$. From $|q| \lesssim p$ it follows, by homogeneity arguments, that $\left|\tilde{\psi}_{j} q_{2}\right| \lesssim \tilde{\psi}_{j} p_{2}$ outside a neighborhood of the null section of $T^{*}\left(\mathbb{R}^{n}\right)$; hence, in view of (3.3),

$$
\left|\left(\left(\tilde{\psi}_{j} q_{2}\right) \circ \chi_{j}^{-1}\right)(y, \eta)\right| \lesssim y_{1}^{2 h}|\eta|^{2}+\eta_{1}^{2}, \quad \forall(y, \eta) \in \mathbb{R}^{2 n},|\eta| \gtrsim 1 .
$$

We can thus apply Lemma 3.2 with $s_{2}=\left(\tilde{\psi}_{j} q_{2}\right) \circ \chi_{j}^{-1}$ and, after composing with $\chi_{j}$, multiplication by $\psi_{j}^{2}$ yields the conclusion.

Let us recall a well known result about Weyl Calculus which is largely used in the sequel (see formula (18.5.6) of [8]).

Lemma 3.4. If $a \in S^{m_{1}}\left(\mathbb{R}^{2 n}\right)$ and $b \in S^{m_{2}}\left(\mathbb{R}^{2 n}\right)$ are classical symbols, then

$$
\sigma_{\text {princ }}\left(a^{w} b^{w}\right)=\sigma_{\text {princ }}\left(a^{w}\right) \sigma_{\text {princ }}\left(b^{w}\right)
$$

and

$\sigma_{\text {sub }}\left(a^{w} b^{w}\right)=\sigma_{\text {princ }}\left(a^{w}\right) \sigma_{\text {sub }}\left(b^{w}\right)+\sigma_{\text {princ }}\left(b^{w}\right) \sigma_{\text {sub }}\left(a^{w}\right)-\frac{i}{2}\left\{\sigma_{\text {princ }}\left(a^{w}\right), \sigma_{\text {princ }}\left(b^{w}\right)\right\}$, where $\sigma_{\mathrm{sub}}(A)$ denotes the subprincipal symbol of any classical operator $A$.

Lemma 3.5. Let $q \sim \sum_{k \geq 0} q_{2-k}$ be as in Theorem 1.1. Then for any $j=1, \ldots, N$,

$$
\sigma\left(F_{j}\left(\psi_{j}^{2} q\right)^{w} F_{j}^{*}\right)-\left(\left(\psi_{j}^{2} q\right) \circ \chi_{j}^{-1}+\left(\psi_{j} \circ \chi_{j}^{-1}\right)\left(\eta_{1} r_{0, j}+y_{1}^{h} r_{1, j}\right)\right) \in S^{0}\left(\mathbb{R}^{2 n}\right),
$$

where $r_{i, j} \in S^{i}\left(\mathbb{R}^{2 n}\right)$ are positively homogeneous symbols of degree $i=0,1$.

Proof. In view of (3.2), one has

$$
\sigma_{\text {princ }}\left(F_{j}\left(\psi_{j}^{2} q\right)^{w} F_{j}^{*}\right)=\left(\psi_{j}^{2} q_{2}\right) \circ \chi_{j}^{-1} .
$$

The proof then follows from a precise description of $\sigma_{\mathrm{sub}}\left(F_{j}\left(\psi_{j}^{2} q\right)^{w} F_{j}^{*}\right)$. To this purpose consider, for $j=1, \ldots, N$, classical operators $A_{i, j} \in \mathrm{OP} S^{i}\left(\mathbb{R}^{n}\right), i=0,1,2$, $\Psi_{j}, U_{j} \in \mathrm{OP} S^{0}\left(\mathbb{R}^{n}\right)$ and $V_{j} \in \mathrm{OP} S^{1}\left(\mathbb{R}^{n}\right)$ with principal symbols $a_{i, j}, \psi_{j}, u_{j}$ and $v_{j}$ respectively $\left(a_{i, j}\right.$ are the symbols defined in Corollary 3.3). Moreover, set

$$
W_{j}=\Psi_{j}^{2} A_{2, j} U_{j}^{2 h}+\Psi_{j}^{2} A_{1, j} V_{j} U_{j}^{h}+\Psi_{j}^{2} A_{0, j} V_{j}^{2}, \quad j=1, \ldots, N,
$$

and note that

$$
\left(\psi_{j}^{2} q\right)^{w}=W_{j}+\left(\left(\psi_{j}^{2} q\right)^{w}-W_{j}\right) .
$$

Upon denoting $l_{j}=\sigma_{\text {princ }}\left(\left(\psi_{j}^{2} q\right)^{w}-W_{j}\right) \in S^{1}\left(\mathbb{R}^{2 n}\right)$, one gets

$$
\psi_{j}^{2} q_{1}=\sigma_{\mathrm{sub}}\left(\left(\psi_{j}^{2} q\right)^{w}\right)=\sigma_{\mathrm{sub}}\left(W_{j}\right)+l_{j} .
$$

Hence, by Lemma 3.4 .

$$
\psi_{j}^{2} q_{1}=-i \frac{h}{2} \psi_{j}^{2} u_{j}^{h-1} a_{1, j}\left\{v_{j}, u_{j}\right\}+l_{j}+\psi_{j}\left(v_{j} \rho_{0, j}+u_{j}^{h} \rho_{1, j}\right),
$$


where $\rho_{i, j} \in S^{i}\left(\mathbb{R}^{2 n}\right)$ are positively homogeneous symbols of degree $i=0,1$. On the other hand, from (3.10) we get

$$
F_{j}\left(\psi_{j}^{2} q\right)^{w} F_{j}^{*}=F_{j} W_{j} F_{j}^{*}+F_{j}\left(\left(\psi_{j}^{2} q\right)^{w}-W_{j}\right) F_{j}^{*} ;
$$

hence

$$
\sigma_{\mathrm{sub}}\left(F_{j}\left(\psi_{j}^{2} q\right)^{w} F_{j}^{*}\right)=\sigma_{\mathrm{sub}}\left(F_{j} W_{j} F_{j}^{*}\right)+l_{j} \circ \chi_{j}^{-1} .
$$

Since the operators $F_{j} W_{j} F_{j}^{*}$ have the same structure of $W_{j}$, and $\chi_{j}$ preserves the Poisson brackets, we can once more use Lemma 3.4 to get

$$
\begin{aligned}
\sigma_{\mathrm{sub}}\left(F_{j} W_{j} F_{j}^{*}\right)= & -i \frac{h}{2}\left(\psi_{j}^{2} u_{j}^{h-1} a_{1, j}\left\{v_{j}, u_{j}\right\}\right) \circ \chi_{j}^{-1} \\
& +\left(\psi_{j} \circ \chi_{j}^{-1}\right)\left(\eta_{1} \tilde{\rho}_{0, j}+y_{1}^{h} \tilde{\rho}_{1, j}\right),
\end{aligned}
$$

where $\tilde{\rho}_{i, j} \in S^{i}\left(\mathbb{R}^{2 n}\right)$ are positively homogeneous symbols of degree $i=0,1$.

Finally, by means of (3.11), (3.12) and (3.13), we obtain

$$
\begin{aligned}
\sigma_{\mathrm{sub}}\left(F_{j}\left(\psi_{j}^{2} q\right)^{w} F_{j}^{*}\right)= & \left(\psi_{j}^{2} q_{1}\right) \circ \chi_{j}^{-1}+\left(\psi_{j} \circ \chi_{j}^{-1}\right)\left(\left(\tilde{\rho}_{0, j}-\rho_{0, j} \circ \chi_{j}^{-1}\right) \eta_{1}\right. \\
& \left.+\left(\tilde{\rho}_{1, j}-\rho_{1, j} \circ \chi_{j}^{-1}\right) y_{1}^{h}\right),
\end{aligned}
$$

whence, by setting $r_{0, j}=\tilde{\rho}_{0, j}-\rho_{0, j} \circ \chi_{j}^{-1}$ and $r_{1, j}=\tilde{\rho}_{1, j}-\rho_{1, j} \circ \chi_{j}^{-1}$, we have

$$
\sigma\left(F_{j}\left(\psi_{j}^{2} q\right)^{w} F_{j}^{*}\right)-\left(\psi_{j}^{2} q_{2}+\psi_{j}^{2} q_{1}\right) \circ \chi_{j}^{-1}-\left(\psi_{j} \circ \chi_{j}^{-1}\right)\left(\eta_{1} r_{0, j}+y_{1}^{h} r_{1, j}\right) \in S^{0}\left(\mathbb{R}^{2 n}\right) .
$$

Since $\psi_{j}^{2}\left(q-q_{2}-q_{1}\right) \in S^{0}\left(\mathbb{R}^{2 n}\right)$, the conclusion easily follows .

The following remark shows that hypothesis (1.8) is invariant under conjugation by Fourier integral operators.

Remark 3.6. Let $p \sim \sum_{k \geq 0} p_{2-k}$ be as in Theorem 1.1 Then, by virtue of (3.3), Lemma 3.2 can be applied to $s_{2}=p_{2} \circ \chi_{j}^{-1}$, and by arguing as in Lemma 3.5 we get, for any $j=1, \ldots, N$,

$\sigma\left(F_{j}\left(\psi_{j}^{2} p\right)^{w} F_{j}^{*}\right)-\left(\psi_{j}^{2} p_{2}\right) \circ \chi_{j}^{-1}-\left(\psi_{j}^{2} p_{1}\right) \circ \chi_{j}^{-1}-\left(\psi_{j} \circ \chi_{j}^{-1}\right)\left(\eta_{1} m_{0, j}+y_{1}^{h} m_{1, j}\right) \in S^{0}\left(\mathbb{R}^{2 n}\right)$, where $m_{i, j} \in S^{i}\left(\mathbb{R}^{2 n}\right)$ are positively homogeneous symbols of degree $i=0,1$.

We now apply the results above to prove Proposition 3.1

Proof of Proposition 3.1. Observe that from (1.10) we have

$$
\left|\psi_{j}^{2} q\right| \leq \psi_{j}^{2} p_{2}+\psi_{j}^{2} p_{1}+\psi_{j}^{2}\left(p-p_{2}-p_{1}\right) \lesssim \psi_{j}^{2} p_{2}+\psi_{j}^{2}\left|p_{1}\right|+\psi_{j}^{2}
$$

By Lemma 3.5, (1.8) and (3.14), we then get

$$
\left|\sigma\left(F_{j}\left(\psi_{j}^{2} q\right)^{w} F_{j}^{*}\right)\right| \lesssim\left(\psi_{j}^{2} p_{2}\right) \circ \chi_{j}^{-1}+\left(\psi_{j} \circ \chi_{j}^{-1}\right)\left(\left|\eta_{1}\right|+\left|y_{1}\right|^{h}|\eta|\right)+1 .
$$

On the other hand, we have

$\left(\psi_{j} \circ \chi_{j}^{-1}\right)\left|\eta_{1}\right| \leq\left(\psi_{j} \circ \chi_{j}^{-1}\right)^{2}\left|\eta_{1}\right|^{2}+1, \quad\left(\psi_{j} \circ \chi_{j}^{-1}\right)\left|y_{1}\right|^{h}|\eta| \leq\left(\psi_{j} \circ \chi_{j}^{-1}\right)^{2}\left|y_{1}\right|^{2 h}|\eta|^{2}+1$, hence, (3.3) and (3.15) yield

$$
\left|\sigma\left(F_{j}\left(\psi_{j}^{2} q\right)^{w} F_{j}^{*}\right)\right| \lesssim\left(\psi_{j}^{2} p_{2}\right) \circ \chi_{j}^{-1}+1,
$$

and this completes the proof. 
Finally we conclude this section by proving Theorem 1.1 .

Proof of Theorem 1.1. As already observed, it is enough to prove inequality (2.1) for each $j=1, \ldots, N$. To this aim, let us define

$$
\tilde{p}_{j}=\left(\psi_{j}^{2} p_{2}\right) \circ \chi_{j}^{-1}+\left(1-\psi_{j}^{2} \circ \chi_{j}^{-1}\right)\left(\eta_{1}^{2}+y_{1}^{2 h}|\eta|^{2}\right),
$$

and note that $0 \leq \tilde{p}_{j} \in S^{2}\left(\mathbb{R}^{2 n}\right)$ is a positively homogeneous symbol behaving like $\eta_{1}^{2}+y_{1}^{2 h}|\eta|^{2}$ near $\left\{(y, \eta) \in \mathbb{R}^{2 n}: y_{1}=\eta_{1}=0, \eta \neq 0\right\}$.

By virtue of Proposition 3.1 we have

$$
\left|\sigma\left(F_{j}\left(\psi_{j}^{2} q\right)^{w} F_{j}^{*}\right)\right| \lesssim \tilde{p}_{j}+1
$$

We can thus apply Theorem 4.1 and Remark 4.2 of Section 4 below, with $L=\tilde{p}_{j}$ to obtain, for any compact set $\tilde{K}$ in $\mathbb{R}^{n}$,

$$
\left\|F_{j}\left(\psi_{j}^{2} q\right)^{w} F_{j}^{*} v\right\|_{0}^{2} \lesssim\left\|\tilde{p}_{j}^{w} v\right\|_{0}^{2}+\|v\|_{0}^{2}, \quad \forall v \in C_{0}^{\infty}(\tilde{K}) .
$$

From Remark 3.6 we conclude that, for every $v \in C_{0}^{\infty}(\tilde{K})$,

$$
\left\|F_{j}\left(\psi_{j}^{2} p\right)^{w} F_{j}^{*} v\right\|_{0}^{2} \geq C_{j}\left\|\left(\left(\psi_{j}^{2} p_{2}\right) \circ \chi_{j}^{-1}\right)^{w} v\right\|_{0}^{2}-c_{j}\left(\left\|t_{j}^{w} v\right\|_{0}^{2}+\|v\|_{0}^{2}\right),
$$

where $t_{j}=\left(\psi_{j}^{2} p_{1}\right) \circ \chi_{j}^{-1}+\left(\psi_{j} \circ \chi_{j}^{-1}\right)\left(\eta_{1} m_{0, j}+y_{1}^{h} m_{1, j}\right) \in S^{1}\left(\mathbb{R}^{2 n}\right)$.

Let us now observe that, by means of (1.8), we have, for every $R \gg 1$,

$$
\left|t_{j}\right| \leq \bar{C}_{j}\left(\psi_{j} \circ \chi_{j}^{-1}\right)\left(\left|\eta_{1}\right|+\left|y_{1}\right|^{h}|\eta|\right) \leq \frac{\bar{C}_{j}}{R}\left(\psi_{j}^{2} \circ \chi_{j}^{-1}\right)\left(\eta_{1}^{2}+y_{1}^{2 h}|\eta|^{2}\right)+R \bar{C}_{j} .
$$

Thus, by using (3.3) we can say that

$$
\left|t_{j}\right| \leq \frac{c_{j}^{\prime} \tilde{p}_{j}}{R}+c_{j}^{\prime} R
$$

with $c_{j}^{\prime}$ independent of $R$.

Theorem 4.3 can be applied to $t_{j}$ and $\tilde{p}_{j}$ yielding

$$
\left\|t_{j}^{w} v\right\|_{0}^{2} \leq \frac{c_{j}^{\prime \prime}}{R}\left\|\tilde{p}_{j}^{w} v\right\|_{0}^{2}+C(R)\|v\|_{0}^{2}, \quad \forall v \in C_{0}^{\infty}(\tilde{K}),
$$

where $c_{j}^{\prime \prime}$ are independent of $R$.

From (3.17) then it follows that, for every $R \gg 1$ and every $v \in C_{0}^{\infty}(\tilde{K})$,

$$
\left\|F_{j}\left(\psi_{j}^{2} p\right)^{w} F_{j}^{*} v\right\|_{0}^{2} \geq C_{j}\left\|\left(\left(\psi_{j}^{2} p_{2}\right) \circ \chi_{j}^{-1}\right)^{w} v\right\|_{0}^{2}-\frac{\tilde{c}_{j}}{R}\left\|\tilde{p}_{j}^{w} v\right\|_{0}^{2}-\tilde{C}(R)\|v\|_{0}^{2},
$$

with $C_{j}$ and $\tilde{c}_{j}$ independent of $R$.

Since $\psi_{j}=1$ on the support of $\varphi_{j}$ one has

$$
\left(\left(1-\psi_{j}^{2} \circ \chi_{j}^{-1}\right)\left(\eta_{1}^{2}+y_{1}^{2 h}|\eta|^{2}\right)\right)^{w} \circ F_{j} \varphi_{j}^{w} F_{j}^{*} \in \mathrm{OP} S^{-\infty} .
$$

Hence

$$
\tilde{p}_{j}^{w} F_{j} \varphi_{j}^{w} F_{j}^{*} F_{j} u=\left(\left(\psi_{j}^{2} p_{2}\right) \circ \chi_{j}^{-1}\right)^{w} F_{j} \varphi_{j}^{w} F_{j}^{*} F_{j} u+L_{j} F_{j} u, \quad L_{j} \in \mathrm{OP} S^{-\infty}\left(\mathbb{R}^{n}\right) .
$$

We now consider (3.16) and (3.19) with $v=F_{j} \varphi_{j}^{w} F_{j}^{*} F_{j} u$, where $u \in C_{0}^{\infty}(K)$, and choose $R$ big enough in (3.16). We get

$$
\left\|F_{j}\left(\psi_{j}^{2} p\right)^{w} F_{j}^{*} F_{j} \varphi_{j}^{w} F_{j}^{*} F_{j} u\right\|_{0}^{2}+\|u\|_{0}^{2} \gtrsim\left\|F_{j}\left(\psi_{j}^{2} q\right)^{w} F_{j}^{*} F_{j} \varphi_{j}^{w} F_{j}^{*} F_{j} u\right\|_{0}^{2} .
$$


Since $F_{j}^{*}$ and $F_{j}$ are 0 -order FIO, applying (3.1) gives, for $j=1, \ldots, N$,

$$
\left\|\left(\psi_{j}^{2} p\right)^{w} \varphi_{j}^{w} u\right\|_{0}^{2}+\|u\|_{0}^{2} \gtrsim\left\|\left(\psi_{j}^{2} q\right)^{w} \varphi_{j}^{w} u\right\|_{0}^{2}, \quad \forall u \in C_{0}^{\infty}(K),
$$

and this concludes the proof.

We complete this section by giving a "rough" sketch of the proof of Theorem 1.1 in the higher codimension case $\nu \geq 1$, i.e. when $\Sigma$ is a symplectic manifold of $T^{*} \mathbb{R}^{n}$ given by the transversal intersection of two involutive cones $\Sigma_{1}$ and $\Sigma_{2}$ of codimension $\nu$. See [12] for further details.

By proceeding as in Proposition 2.3, we can prove that, for every point $\rho$ of $\Sigma$, there exist a conic neighborhood $U$ of $\varrho$ in $T^{*} \mathbb{R}^{n} \backslash 0$, a conic neighborhood $V$ in $T^{*} \mathbb{R}^{n} \backslash 0$ and a canonical symplectomorphism $\chi: U \longrightarrow V$ for which $\chi\left(U \cap \Sigma_{1}\right)=$ $\left\{(y, \eta) \in V \mid y_{1}=\ldots=y_{\nu}=0\right\}$ and $\chi\left(U \cap \Sigma_{2}\right)=\left\{(y, \eta) \in V \mid \eta_{1}=\ldots=\eta_{\nu}=0\right\}$. In doing so, it is crucial that $\Sigma_{1}$ and $\Sigma_{2}$ are involutive submanifolds of $T^{*} \mathbb{R}^{n}$.

By (1.7) and by using the FIO associated with $\chi$, we reduce $p^{w}$ to a Grushin-type operator with principal symbol $p_{2} \circ \chi^{-1}(y, \eta) \approx \sum_{j=1}^{\nu}\left(\eta_{j}^{2}+|\eta|^{2} y_{j}^{2 h}\right)$. At this point Proposition 3.1 is easily proved in the higher codimension case, by repeating the same steps worked out previously for $\nu=1$; whereas, the assertion in Remark 3.6 has to be adapted and replaced by

$$
\begin{aligned}
& \sigma\left(F_{j}\left(\psi_{j}^{2} p\right)^{w} F_{j}^{*}\right)-\left(\psi_{j}^{2} p_{2}\right) \circ \chi_{j}^{-1}-\left(\psi_{j}^{2} p_{1}\right) \circ \chi_{j}^{-1} \\
& -\left(\psi_{j} \circ \chi_{j}^{-1}\right)\left(\sum_{k=1}^{\nu} \eta_{k} m_{0, k, j}+\sum_{\alpha \in \mathbb{Z}_{+}^{\nu},|\alpha|=h} y^{\alpha} m_{1, \alpha, j}\right) \in S^{0}\left(\mathbb{R}^{2 n}\right),
\end{aligned}
$$

where $m_{0, k, j}$ and $m_{1, \alpha, j}$ are positively homogeneous symbols of degree 0,1 , respectively.

Henceforth the proof of Theorem 1.1 follows similarly to the case $\nu=1$.

\section{Appendix}

In 11] we proved Fefferman's SAK Principle for certain second order operators. More precisely, if $L \in S^{2}\left(\mathbb{R}^{2 n}\right)$ is a homogeneous non negative symbol behaving like $\eta_{1}^{2}+|\eta|^{2} y_{1}^{2 h}$ near its characteristic manifold $\left\{(y, \eta) \in \mathbb{R}^{2 n}: \eta_{1}=y_{1}=0, \eta \neq 0\right\}$, the following theorem holds.

Theorem 4.1. Let $q \in S^{2}\left(\mathbb{R}^{2 n}\right)$ satisfy, for some positive constant $\delta$, the condition $|q(y, \eta)| \leq \delta L(y, \eta)$, for every $(y, \eta) \in \mathbb{R}^{2 n}$. Then for every $K \subset \subset \mathbb{R}^{n}$ there exist positive constants $C$, $c$ such that

$$
\left\|q^{w} v\right\|_{0}^{2} \leq C\left\|L^{w} v\right\|_{0}^{2}+c\|v\|_{0}^{2}, \quad \forall v \in C_{0}^{\infty}(K) .
$$

Actually, in [11] we showed that (4.1) holds under a weaker assumption: it suffices that $\max _{B_{i}}|q| \leq \delta \max _{B_{i}} L$ where $\left\{B_{i}\right\}_{i \in \mathcal{J}}$ is a suitable partition of $\mathbb{R}_{y}^{n} \times \mathbb{R}_{\eta}^{n}$ (see also [16]).

Remark 4.2. In Theorem 4.1 we can replace the hypothesis by $|q(y, \eta)| \leq \delta L(y, \eta)+$ $\delta^{\prime}$, with $\delta, \delta^{\prime}$ positive constants, since the right hand side of inequality (4.1) is invariant under $L^{2}\left(\mathbb{R}^{n}\right)$-perturbations.

A slight modification of the arguments developed in [11] allows, in a special case, a better control on the constant $C$ in (4.1). Namely, if $q \in S^{1}\left(\mathbb{R}^{2 n}\right)$ one can prove that the constant $C$ in (4.1) can be chosen small if $\delta$ in Theorem 4.1 is small enough. More precisely the following theorem holds. 
Theorem 4.3. Let us consider $q \in S^{1}\left(\mathbb{R}^{2 n}\right)$ such that

$$
|q(y, \eta)| \leq \frac{c^{\prime}}{R} L(y, \eta)+c^{\prime} R, \quad \forall(y, \eta) \in \mathbb{R}^{2 n},
$$

where $R$ is a large positive parameter and $c^{\prime}$ is a real positive constant independent of $R$.

Then, for every $K \subset \subset \mathbb{R}^{n}$ there exist positive constants $C$ and $c$, with $C$ independent of $R$, such that

$$
\left\|q^{w} v\right\|_{0}^{2} \leq \frac{C}{R^{2}}\left\|L^{w} v\right\|_{0}^{2}+c\|v\|_{0}^{2}, \quad \forall v \in C_{0}^{\infty}(K) .
$$

Sketch of the proof. One uses the techniques developed in [11] to obtain operator estimates from the pointwise comparison between the symbols $R q$ and $p$ defined by

$$
p(y, \eta)=\chi(y) L(y, \eta)+(1-\chi(y))\left(\eta_{1}^{2}+y_{1}^{2 h}\left|\eta^{\prime}\right|^{2}\right),
$$

$\chi \in C_{0}^{\infty}\left(\mathbb{R}^{n}\right), \chi=1$, on a neighborhood of $K$ with $0 \leq \chi \leq 1$.

As in [11] we construct a suitable partition $\left\{Q_{\mu}\right\}_{\mu}$ of the phase space $\mathbb{R}_{y}^{n} \times \mathbb{R}_{\eta}^{n}$ in rectangles $Q_{\mu}$ centered in $\left(y_{\mu}, \eta_{\mu}\right)$, and we prove that the estimate (4.2) can be microlocalized in these rectangles $Q_{\mu}$.

We distinguish between two cases: the rectangles for which $\left|\eta_{\mu}\right| \geq 2 R$ (type 1) and those for which $\left|\eta_{\mu}\right| \leq 2 R$ (type 2).

The operators $(R q)^{w}$ and $p^{w}$ microlocalized in the rectangles of type 2 are $L^{2}$ continuous maps yielding negligible errors in the estimates. On the other hand, when we microlocalize the same operators in the rectangles of type 1 , we observe that the seminorms of the related symbols are independent of $\mu$ and $R$. This allows us to control the dependance on $R$ of the constants in the corresponding microlocal estimates.

By patching together the microlocal estimates, we achieve

$$
\left\|R q^{w} v\right\|_{0}^{2} \leq C\left\|p^{w} v\right\|_{0}^{2}+\tilde{c}\|v\|_{0}^{2}, \quad \forall v \in C_{0}^{\infty}(K)
$$

with $C$ independent of $R$.

\section{ACKNOWLEDGEMENTS}

The authors express their gratitude to A. Parmeggiani for introducing them to the argument and for the useful discussions.

\section{REFERENCES}

1. R.Beals, C.L.Fefferman: On local solvability of linear partial differential equations. Ann. of Math., 97, 482-498 (1974). MR0352746 (50:5233)

2. L.Boutet de Monvel, A.Grigis and B.Helffer: Paramétrixes d'opérateurs pseudo-différentiels à caractéristiques multiples. Astérisque, 34-35, 93-121 (1976). MR0493005 (58:12046)

3. C.L.Fefferman, D.H.Phong: On positivity of pseudo-differential operators. Proc. Nat. Acad. Sci., 75, 4673-4674 (1978). MR0507931 (80b:47064)

4. C.L.Fefferman: The Uncertainty Principle. Bull. A.M.S., 9, 129-206 (1983). MR0707957 (85f:35001)

5. D.Fujiwara: A construction of approximate positive parts of essentially selfadjoint pseudodifferential operators. Commun. Pure Appl. Math., 37, 101-147 (1984). MR0728268 (85i:47051)

6. F.Hérau: Fefferman's SAK principle in one dimension. Ann. Inst. Fourier, 50 (4), 1229-1264 (2000). MR:1799744 (2001k:35310)

7. L.Hörmander: The Cauchy problem for differential equations with double characteristics. J. d'Analyse Mathématique, 32, 118-196, (1977). MR0492751(58:11822) 
8. L.Hörmander: The Analysis of Linear Partial Differential Operators, Vol. III and Vol. IV. Springer-Verlag (1983/85). MR0781536 (87d:35002a) MR0781537(87d:35002b)

9. R.Lascar: Propagation des singularites et hypoellipticite pour des operateurs pseudodifferentiels a caracteristiques doubles. Comm. in Partial Differential Equations, 3 (3), 201-247 (1978). MR0492798 (58:11863)

10. N.Lerner, J.Nourrigat: Lower bounds for pseudo-differential operators. Ann. Inst. Fourier, 40 (3), 657-682 (1990). MR1091836 (92a:35172)

11. L.Maniccia, M.Mughetti: SAK principle for a class of Grushin-type operators. Revista Matematicá Iberoamericana, 22 (1), 259-286 (2006). MR2268119

12. L.Maniccia, M.Mughetti: Fefferman's SAK principle and a priori estimates for second order operators. Ann. Univ. Ferrara Sez. VII Sci. Mat., 52, 337-352 (2007). MR2273103

13. S.Mustapha: Sous ellipticité dans le cadre du calcul $S(m, g)$. Comm. Partial Differential Equations 19 (1-2), 245-275 (1994). MR1257005 (95c:35279)

14. S.Mustapha: Sous-ellipticité dans le cadre du calcul $S(m, g)$. II. Comm. Partial Differential Equations 20 (3-4), 541-566 (1995). MR1318080 (96a:35225)

15. A.Parmeggiani: A Class of counterexamples to the Fefferman-Phong Inequality for systems. Comm. in Partial Differential Equations, 29 (9,10), 1281-1303 (2004). MR 2103837 (2005h:35385)

16. A.Parmeggiani: Subunit balls for symbols of pseudodifferential operators. Adv. Math., 131(2), 357-452 (1997). MR1483973 (99c:35270)

17. D.Tataru: On the Fefferman-Phong inequality and related problems. Comm. in Partial Differential Equations, 27 (11,12), 2101-2138 (2002). MR.1944027 (2003m:35259)

18. F.Treves: Introduction to Pseudodifferential and Fourier Integral Operators, Vol. II. Plenum Press (1980). MR0597145 (82i:58068)

Department of Mathematics, University of Bologna, Piazza di Porta S.Donato 5, 40127 Bologna, ITALY

E-mail address: maniccia@dm.unibo.it

Department of Mathematics, University of Bologna, Piazza di Porta S.Donato 5, 40127 Bologna, ItAly

E-mail address: mughetti@dm.unibo.it 